\title{
RETRACTED ARTICLE: Role of TLR2 and TLR4 Signaling in Parkinson's Disease: An Insight into Associated Therapeutic Potential
}

\author{
Gagandeep Kaur ${ }^{1} \cdot$ Tapan Behl $^{1}{ }^{10} \cdot$ Aayush Sehgal $^{1}$ - Sukhbir Singh ${ }^{1} \cdot$ Neelam Sharma $^{1} \cdot$ Arun Kumar $^{1}$. \\ Sandeep Arora ${ }^{1} \cdot$ Simona Bungau ${ }^{2}$
}

Received: 5 December 2020 / Accepted: 2 February 2021 / Published online: 9 March 2021

(C) The Author(s), under exclusive licence to Springer Science+Business Media, LLC part of Springer Nature 2021

The authors have retracted this article because it contains material that overlaps with content from a previously published article by different authors [1]. All authors agree to this retraction. The online version of this article contains the full text of the retracted article as Supplementary Information.
Supplementary Information The online version contains supplementary material available at https://doi.org/10.1007/s12031-021-01811-z.

\section{Reference}

Kouli, Antonina, C. B. Horne, and C. H. Williams-Gray. "Toll-like receptors and their therapeutic potential in Parkinson's disease and $\alpha$-synucleinopathies." Brain, behavior, and immunity 81 (2019): 41-51. https://doi.org/10.1016/j.bbi.2019.06.042

The online version of this article contains the full text of the retracted article as Supplementary Information: https://doi.org/10. 1007/s12031-021-01811-z

Tapan Behl

tapanbehl31@gmail.com; tapan.behl@chitkara.edu.in

1 Chitkara College of Pharmacy, Chitkara University, Punjab, India

2 Department of Pharmacy, Faculty of Pharmacy, University of Oradea, Oradea, Romania 